\title{
INI1 expression is retained in composite rhabdoid tumors, including rhabdoid meningiomas
}

\author{
Arie Perry ${ }^{1}$, Christine E Fuller ${ }^{2}$, Alexander R Judkins ${ }^{3}$, Louis P Dehner ${ }^{1}$ and \\ Jaclyn A Biegel ${ }^{3}$ \\ ${ }^{1}$ Department of Pathology, Washington University School of Medicine, St Louis, MO, USA; ${ }^{2}$ Department of \\ Pathology, St Jude Children's Research Hospital, Memphis, TN, USA and ${ }^{3}$ Departments of Pathology and \\ Pediatrics, Children's Hospital of Philadelphia and University of Pennsylvania School of Medicine, \\ Philadelphia, PA, USA
}

\begin{abstract}
Rhabdoid cells are encountered in specific entities, such as malignant rhabdoid tumor and atypical teratoid/ rhabdoid tumor, as well as in composite rhabdoid tumors derived secondarily from other tumor types. Although rhabdoid tumors are uniformly aggressive, distinction of the entity from the phenotype remains important for its therapeutic implications. The majority of malignant rhabdoid tumors and atypical teratoid/rhabdoid tumors affect infants and young children, harbor chromosome 22q deletions, and inactivate the INI1/hSNF5/BAF47 tumor suppressor gene on 22q11.2. In contrast, most composite rhabdoid tumors are diagnosed in adults, with FISH detectable 22q losses the exception rather than the rule. However, this assay remains limited since 22q dosages are maintained in $\mathbf{2 0}-30 \%$ of malignant rhabdoid tumors and atypical teratoid/rhabdoid tumors. Furthermore, chromosome 22 losses are common in some parent tumor types, particularly meningiomas. The recently developed INI1 antibody shows loss of nuclear expression in malignant rhabdoid tumors and atypical teratoid/rhabdoid tumors, though its status in composite rhabdoid tumors is largely unknown. Therefore, we utilized immunohistochemistry and FISH to study INI1 expression and 22q dosages, respectively, in 40 composite rhabdoid tumors, including 16 meningiomas, 15 carcinomas, three melanomas, two sarcomas, two glioblastomas, and 1 neuroblastoma. Approximately $70 \%$ of rhabdoid meningiomas had a $22 q$ deletion, but this was rare in other tumor types. Except for one retroperitoneal leiomyosarcoma, nuclear INI1 expression was retained in all composite rhabdoid tumors, including meningiomas with 22q deletion. Therefore, we conclude that INI1 immunohistochemistry is a relatively simple, sensitive, and specific technique for distinguishing malignant rhabdoid tumor and atypical teratoid/rhabdoid tumor from composite rhabdoid tumor.
\end{abstract}

Modern Pathology (2005) 18, 951-958. doi:10.1038/modpathol.3800375; Published online 11 March 2005

Keywords: atypical teratoid/rhabdoid tumor; differential diagnosis; FISH; immunohistochemistry; INI1; malignant rhabdoid tumor; rhabdoid phenotype

The designation of a neoplasm as 'rhabdoid' in type relies on the presence of large epithelioid cells with eccentric eosinophilic cytoplasm, vesicular nuclei, prominent nucleoli, and globular/fibrillar paranuclear inclusions corresponding ultrastructurally to whorled bundles of intermediate filaments. Even in the absence of the paranuclear inclusion though, the cytologic features are sufficiently characteristic to raise the possibility of a rhabdoid neoplasm. These

Correspondence: Dr A Perry, MD, Division of Neuropathology, Campus Box 8118, Washington University School of Medicine, 660 S. Euclid Ave., St Louis, MO 63110-1093, USA.

E-mail: aperry@wustl.edu

Received 15 November 2004; revised 8 December 2004; accepted 9 December 2004; published online 11 March 2005 cellular findings were initially described in malignant rhabdoid tumor of the kidney ${ }^{1,2}$ and were designated 'rhabdoid' based on their resemblance to rhabdomyoblasts, but lack of ultrastructural evidence for skeletal muscle differentiation. This cell type was subsequently identified in many extrarenal sites and tumor types, leading to considerable debate regarding issues of nomenclature and histogenesis. ${ }^{3-7}$ However, accumulating data suggest that rhabdoid tumors exist as both a specific entity and a secondary morphologic phenotype encountered within a wide array of tumor types, typically signifying the emergence of cytologic anaplasia, high-grade features, and aggressive biology. The two generally accepted diagnostic entities are malignant rhabdoid tumor (renal and extrarenal 
forms) and atypical teratoid/rhabdoid tumor of the central nervous system..$^{2,8-14}$ Both malignant rhabdoid tumor and atypical teratoid/rhabdoid tumor have a distinct predilection for infants and young children (including congenital and disseminated presentations), a highly aggressive biology with short survival times, a polyphenotypic immunoprofile, and characteristic deletions and mutations (somatic or germline) involving the INI1/ hSNF5 tumor suppressor gene on chromosome 22q11.2. ${ }^{11,15-24}$ Although the histogenesis is unknown, it is likely that the malignant rhabdoid tumor and atypical teratoid/rhabdoid tumor represent the same basic neoplasm, differing only in the appellation assigned for different sites of origin. The secondary rhabdoid phenotype is most often found in neoplasms of adults and has been encountered in a variety of parent neoplasms, including carcinomas, melanomas, sarcomas, desmoplastic small round cell tumors, neuroblastomas, meningiomas, and gliomas. ${ }^{7,25-34}$ Occasionally, the parent tumor is not immediately recognizable so that a specific diagnosis is not attainable, beyond that of a highgrade malignant neoplasm. Previously referred to as composite extrarenal rhabdoid tumors, the 'extrarenal' portion is no longer appropriate, given the recent recognition of a rhabdoid variant of renal cell carcinoma. ${ }^{27,33}$ Therefore, we currently refer to them simply as composite rhabdoid tumors.

For reasons that are poorly understood, rhabdoid cytology is nearly universally associated with aggressive behavior. A recent study suggested that loss of INI1/hSNF5 function affects the actin cytoskeleton, providing a potential explanation for the rhabdoid morphology itself. ${ }^{35}$ Another study suggests that cytokeratin 8 gene (KRT 8) mutations result in the formation of intracytoplasmic intermediate filament inclusions. ${ }^{36}$ Therefore, it is possible that the rhabdoid morphology may arise through similar molecular mechanisms regardless of the histogenesis. Nevertheless, distinguishing composite rhabdoid tumors from malignant rhabdoid tumors and atypical teratoid/rhabdoid tumors remains important given differences in therapeutic approach. For the former, patient management typically follows the guidelines of the parent neoplasm, whereas for the latter, an extremely aggressive protocol with high-dose chemotherapy and stem cell rescue has been advocated. ${ }^{14}$ In most examples, the clinicopathologic features are sufficiently specific to clearly distinguish one from the other (Table 1). Using immunohistochemistry, the majority of malignant rhabdoid tumors and atypical teratoid/rhabdoid tumors have a polyphenotypic immunoprofile that includes epithelial membrane antigen (EMA), vimentin, smooth muscle actin, and CD99 positivity, as well as variable immunoreactivities for cytokeratins, glial fibrillary acidic protein (GFAP), synaptophysin, neurofilament, S-100 protein, and desmin. In contrast, most composite rhabdoid tumors retain the same or a slightly altered immunoprofile of the parent neoplasm. However, there remains sufficient clinical, morphologic, and immunohistochemical overlap such that a subset of cases are problematic. This is further complicated by the fact that PNET-like, carcinoma-like, and sarcoma-like foci are all common in malignant rhabdoid tumors and atypical teratoid/rhabdoid tumors, occasionally predominating over the rhabdoid cells. We have previously shown that FISH analysis for the detection of 22q deletions is a useful technique in this differential diagnosis and is applicable to formalin-fixed paraffin-embedded tissue. ${ }^{7,9,20}$ Nevertheless, this assay is limited by the fact that it is not yet widely available, $20-30 \%$ of malignant rhabdoid tumors and atypical teratoid/ rhabdoid tumors have no detectable deletions, and occasional composite rhabdoid tumors harbor deletions. ${ }^{7}$ The latter is particularly relevant for parent tumors that normally have a high frequency of $22 q$ deletions, such as meningioma.

Recently, a commercial antibody for the INI1/ BAF47 protein has become available and shows widespread nuclear positivity in normal cells, including endothelial cells and lymphocytes. The latter thus provide a useful internal control. Loss of nuclear expression has been universally encountered in malignant rhabdoid tumor and atypical

Table 1 Features favoring malignant rhabdoid tumor or atypical teratoid/rhabdoid tumor vs composite rhabdoid tumor

\begin{tabular}{lll}
\hline Parameter & $\begin{array}{l}\text { Malignant rhabdoid tumor or atypical teratoid/ } \\
\text { rhabdoid tumor }\end{array}$ & Composite rhabdoid tumor \\
\hline $\begin{array}{l}\text { Patient age } \\
\text { Location of tumor }\end{array}$ & $\begin{array}{l}\text { Child (<3 years) } \\
\text { Kidney, brain, soft tissue } \\
\text { Histology } \\
\text { Immunohistochemistry } \\
\text { Polyphenotypic profile, loss of INI1 expression }\end{array}$ & $\begin{array}{l}\text { Adult } \\
\text { Extrarenal visceral organs, dura, skin } \\
\text { Recognizable parent neoplasm } \\
\text { Single lineage profile, retained INI1 }\end{array}$ \\
$\begin{array}{l}\text { Ultrastructure } \\
\text { Genetics }\end{array}$ & $\begin{array}{l}\text { Lack of differentiation } \\
\text { 22q deletions common, INI1 mutations, homozygous } \\
\text { deletions }\end{array}$ & $\begin{array}{l}\text { Differentiation consistent with parent neoplasm } \\
\text { 22q deletions uncommon, genetic features of } \\
\text { parent neoplasm }\end{array}$ \\
\hline
\end{tabular}

\footnotetext{
${ }^{\mathrm{a}}$ Based on data from current study.
} 
teratoid/rhabdoid tumor, but not in the majority of other pediatric central nervous system (CNS) and soft tissue tumors. ${ }^{23,24}$ However, this marker has not yet been applied to composite rhabdoid tumors, where it may be particularly useful as an ancillary diagnostic aid. It would also potentially serve a second role in clarifying a basic biologic question for rhabdoid neoplasms. One of the hypotheses states that rhabdoid cytology represents a common endstage pattern in a histogenetically diverse group of tumors. If that is true, then loss of INI1 expression could conceivably represent the common substrate. Therefore, in the current study, we performed INI1 immunohistochemistry on 40 archival, paraffinembedded composite rhabdoid tumors derived from 16 meningiomas, 15 carcinomas, three melanomas, two sarcomas, two glioblastomas, and one neuroblastoma. Our data suggest that composite rhabdoid tumors are genetically distinct from malignant rhabdoid tumor and atypical teratoid/rhabdoid tumor and retain INI1 expression in the majority of cases, including meningiomas and other tumors with chromosome $22 \mathrm{q}$ deletions.

\section{Materials and methods}

The surgical files of the Lauren V Ackerman Laboratory of Surgical Pathology and consultation files of two of the authors (AP, LPD) were searched for the term 'rhabdoid' within the diagnostic line of cases signed out between 1994 and 2004. Those cases arising within a recognizable parent neoplasm (ie composite rhabdoid tumors) were retrieved for further study. A representative paraffin block was cut at $5 \mu \mathrm{m}$ onto positively charged glass slides for immunohistochemistry and FISH analysis. In consult cases lacking an available paraffin block, archived unstained sections were utilized if available.

Immunohistochemistry was performed as previously published, ${ }^{23,24}$ utilizing the BAF47/SNF5 mouse monoclonal antibody (BD Transduction Labs, San Diego, CA, USA) and DAKO autostainer ${ }^{\circledR}$ (Carpinteria, CA, USA). Slides were subjected to heat-induced epitope retrieval pretreatment in citrate buffer ( $\mathrm{pH}$ 6.0) for $3 \mathrm{~min}$, followed by cooling to room temperature. Sections were incubated with primary antibody at a 1:40 dilution for $30 \mathrm{~min}$ at room temperature. Detection was performed utilizing the DAKO Envision Plus HRP secondary antimouse antibody, 3,3-diaminobenzidine (DAB), and counterstaining with hematoxylin.

FISH analysis was performed as previously published $^{7}$ using DNA probes for BCR on 22q11.2 (SpectrumGreen-labeled; Vysis, Inc., Downers Grove, IL, USA) and NF2 on 22q12 (rhodaminelabeled; paired cosmid probes n3022 and n24f20, UK HGMP Resource Centre, http://www.hgmp. mrc.ac.uk; gift from Dr Mia MacCollin, Massachusetts General Hospital, Boston, MA, USA). The BCR probe is located within $0.5 \mathrm{Mb}$ of the INI1 gene and is known to be codeleted in the majority of cases of malignant rhabdoid tumor and atypical teratoid/ rhabdoid tumor. Sections were deparaffinized, steamed in $10 \mathrm{mM}$ citrate buffer, $\mathrm{pH}$ 6.0, and pepsin digested. Paired probes were codiluted to concentrations of 1:25 in DenHyb buffer (Insitus Laboratories, Albuquerque, NM, USA) and $10 \mu \mathrm{l}$ was applied to each slide. Target and probe DNA were codenatured at $90^{\circ} \mathrm{C}$ for $13 \mathrm{~min}$. Hybridization was carried out via overnight incubation at $37^{\circ} \mathrm{C}$ in a humidified oven and the following day, the slides were washed with $50 \%$ formamide/ $1 \times$ SSC, followed by two washes in $2 \times$ SSC for 5 min each. Nuclei were counterstained with DAPI and fluorescent signals were enumerated under an Olympus BX60 fluorescent microscope with appropriate filters (Olympus; Melville, NY, USA). For each hybridization, 100 nonoverlapping nuclei were assessed for numbers of green and red signals. Cutoffs for BCR and NF2 deletions were each set at $50 \%$ nuclei with one signal (mean plus 3 s.d. for non-neoplastic control nuclei with one signal). Hybridizations were considered noninformative if the FISH signals were either lacking or too weak to interpret.

\section{Results}

Clinicopathologic, immunohistochemical, and available genetic data on the 40 cases of composite rhabdoid tumor are summarized in Table 2. A total of 12 cases were published in a prior study. ${ }^{7}$ The parent neoplasms included 16 meningiomas, 15 carcinomas, three melanomas, two sarcomas, two glioblastomas, and one neuroblastoma. The 23 female and 17 male patients ranged in age from 3 months to 80 years (median 60 years) and included 4 children $(<18$ years of age).

By immunohistochemistry, 38 of 39 (97\%) informative cases showed retention of nuclear INI1 expression (Table 2; Figure 1a-f). Although there was regional variability in staining intensities, foci of strong tumoral and/or nontumoral nuclear staining were seen in nearly all tested cases. The three cases considered noninformative had lack of staining within endothelial cells or lymphocytes, or variable expressivity. One retroperitoneal leiomyosarcoma showed loss of INI1 expression in tumor nuclei, with appropriate staining of intratumoral endothelial cells serving as the internal control (Table 2; Figure 1g,h).

Deletions of $22 q$ were identified in 11 of $34(32 \%)$ cases assessed by FISH (Table 2, Figure 2). All but one of the cases with $22 \mathrm{q}$ deletions were meningiomas, with rhabdoid meningiomas showing $22 \mathrm{q}$ deletion in 10 of $14(71 \%)$ cases overall. The 23 nondeleted cases of composite rhabdoid tumor harbored either normal 22q dosages or gains in copy numbers (polysomy) (Table 2, Figure 2). None 
Table 2 Summary of clinicopathologic, immunohistochemical and FISH data

\begin{tabular}{|c|c|c|c|c|c|}
\hline Case & Age/sex & Parent tumor & Organ & $22 q \mathrm{FISH}$ & INI1 \\
\hline 1 & $3 \mathrm{mo} / \mathrm{M}$ & Angiosarcoma & Soft tissue & Normal & Retained \\
\hline $2^{\mathrm{a}}$ & $64 / \mathrm{M}$ & Carcinoma & Lung & Polysomy & Retained \\
\hline $3^{a}$ & $54 / \mathrm{M}$ & Carcinoma & Small bowel & Deleted & Retained \\
\hline $4^{\mathrm{a}}$ & $42 / \mathrm{M}$ & Carcinoma & Kidney & Polysomy & NI \\
\hline $5^{\mathrm{a}}$ & $72 / \mathrm{F}$ & Carcinoma & Uterus & Polysomy & Retained \\
\hline $6^{\mathrm{a}}$ & $68 / \mathrm{F}$ & Carcinoma & Kidney & Normal & Retained \\
\hline $7^{\mathrm{a}}$ & $66 / F$ & Carcinoma (lung) & Femoral metastasis & Normal & Retained \\
\hline 8 & $41 / \mathrm{F}$ & Carcinoma & Liver & ND & Retained \\
\hline 9 & $52 / \mathrm{F}$ & Carcinoma & Uterus & Normal & Retained \\
\hline 10 & $79 / F$ & Carcinoma & Kidney & Normal & Retained \\
\hline 11 & $66 / \mathrm{M}$ & Carcinoma & Kidney & Normal & Retained \\
\hline 12 & $68 / \mathrm{F}$ & Carcinoma & Kidney & Normal & Retained \\
\hline 13 & $68 / \mathrm{F}$ & Carcinoma & Kidney & Polysomy & Retained \\
\hline 14 & $53 / \mathrm{F}$ & Carcinoma & Lung & Polysomy & Retained \\
\hline 15 & $57 / \mathrm{M}$ & Carcinoma & Kidney & Polysomy & Retained \\
\hline 16 & $60 / \mathrm{M}$ & Carcinoma & Kidney & $\mathrm{NI}$ & Retained \\
\hline 17 & $36 / \mathrm{M}$ & Glioblastoma & Brain & ND & NI \\
\hline 18 & $20 / \mathrm{F}$ & Glioblastoma & Brain & ND & NI \\
\hline 19 & $29 / \mathrm{M}$ & Leiomyosarcoma & Soft tissue & Normal & Lost \\
\hline 20 & $80 / \mathrm{M}$ & Melanoma (skin) & Lung metastasis & Normal & Retained \\
\hline $21^{\mathrm{a}}$ & $46 / \mathrm{F}$ & Melanoma & Skin & Polysomy & Retained \\
\hline $22^{\mathrm{a}}$ & $21 / \mathrm{M}$ & Melanoma & Skin & Polysomy & Retained \\
\hline $23^{\mathrm{a}}$ & $60 / \mathrm{F}$ & Melanoma (skin) & Bone metastasis & Polysomy & Retained \\
\hline 24 & $78 / \mathrm{M}$ & Meningioma & Brain & ND & Retained \\
\hline $25^{\mathrm{a}}$ & $65 / \mathrm{M}$ & Meningioma & Brain & Deleted & Retained \\
\hline 26 & $61 / \mathrm{F}$ & Meningioma & Brain & Deleted & Retained \\
\hline $27^{\mathrm{a}}$ & $65 / \mathrm{F}$ & Meningioma & Brain & Normal & Retained \\
\hline 28 & $36 / \mathrm{M}$ & Meningioma & Brain & Deleted & Retained \\
\hline 29 & $68 / \mathrm{F}$ & Meningioma & Brain & Deleted & Retained \\
\hline 30 & $42 / \mathrm{F}$ & Meningioma & Brain & Deleted & Retained \\
\hline 31 & $37 / \mathrm{F}$ & Meningioma & Brain & Normal & Retained \\
\hline 32 & $79 / \mathrm{F}$ & Meningioma & Brain & Deleted & Retained \\
\hline 33 & $52 / \mathrm{F}$ & Meningioma & Brain & Deleted & Retained \\
\hline 34 & $23 / \mathrm{M}$ & Meningioma & Brain & Normal & Retained \\
\hline 35 & 8/M & Meningioma & Brain & Normal & Retained \\
\hline 36 & $59 / \mathrm{F}$ & Meningioma & Brain & Deleted & Retained \\
\hline 37 & $60 / \mathrm{F}$ & Meningioma & Brain & ND & Retained \\
\hline 38 & $15 / \mathrm{F}$ & Meningioma & Brain & Deleted & Retained \\
\hline 39 & $31 / \mathrm{F}$ & Meningioma & Brain & Deleted & Retained \\
\hline $40^{\mathrm{a}}$ & $7 \mathrm{mo} / \mathrm{M}$ & Neuroblastoma & Adrenal & Normal & Retained \\
\hline
\end{tabular}

${ }^{\mathrm{a}}$ Cases previously published in Fuller et al. ${ }^{7}$

$\mathrm{NI}=$ noninformative; $\mathrm{ND}=$ not done. Age is expressed in years except in two instances mentioned with mo. mo $=$ months.

of the 11 cases with 22q deletion had a concomitant loss of INI1 expression. Similarly, the single case of leiomyosarcoma with loss of protein expression showed no associated $22 \mathrm{q}$ deletion by FISH. Mutation screening of paraffin-embedded tissue from this case was attempted, but was unsuccessful.

\section{Discussion}

Despite the rarity of malignant rhabdoid tumor and atypical teratoid/rhabdoid tumor, these aggressive tumors of infants and young children have engendered considerable interest with much progress made over the last 5-10 years in elucidating the biology and genetics. ${ }^{4-36}$ Although the histogenesis remains uncertain, these tumors are generally considered to be a form of primitive or embryonal neoplasm with a distinctive polyphenotypic immunoprofile, usually including combinations of EMA, vimentin, smooth muscle actin, and CD99 expression. Nevertheless, given their remarkably wide morphologic and immunohistochemical spectrum with varying numbers of rhabdoid cells in any one tumor, they can be diagnostically challenging. Composite rhabdoid tumors are similarly challenging, since

Figure 1 Examples of H\&E (a, $\mathbf{c}, \mathbf{e}, \mathbf{g})$ and INI1 $(\mathbf{b}, \mathbf{d}, \mathbf{f}, \mathbf{h})$-stained sections from composite rhabdoid tumors derived from a lung carcinoma $(\mathbf{a}, \mathbf{b})$, a renal cell carcinoma $(\mathbf{c}, \mathbf{d})$, a meningioma $(\mathbf{e}, \mathbf{f})$ and a leiomyosarcoma $(\mathbf{g}, \mathbf{h})$. Nuclear INI1 expression was retained in the first three of these $(\mathbf{b}, \mathbf{d}, \mathbf{f})$. In contrast, there was loss of expression in the leiomyosarcoma, with appropriate staining of intratumoral endothelial cell nuclei (h). 


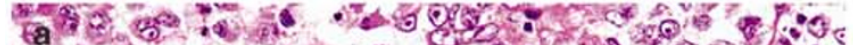

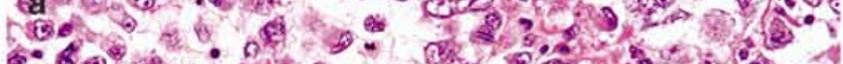

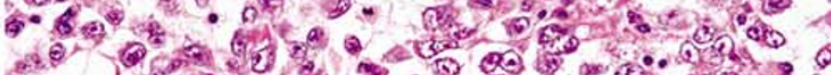

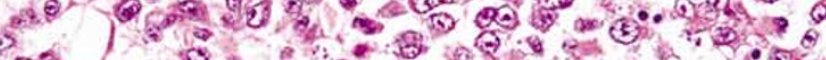

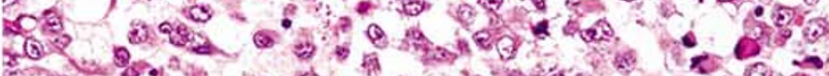

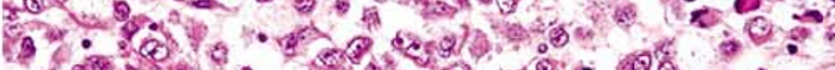

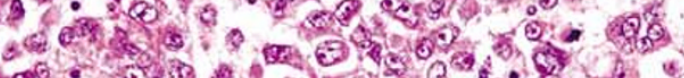

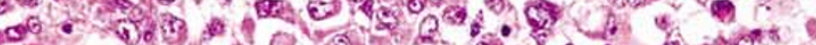

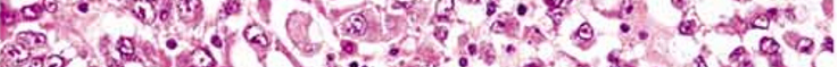

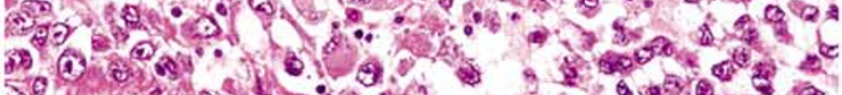

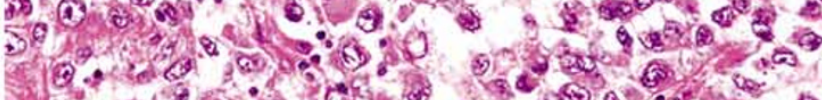

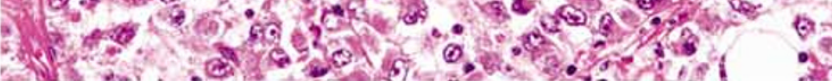

GQ 1 \%

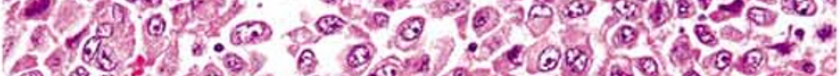
3. 8 1

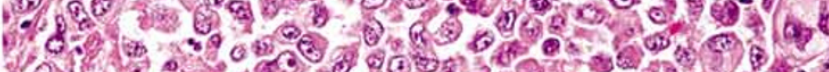

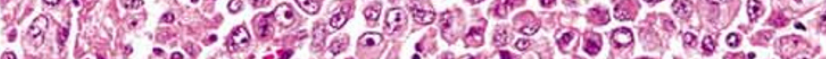
1. $190 \%$ \%

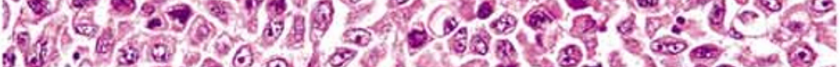

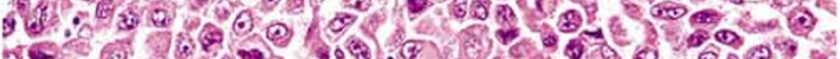

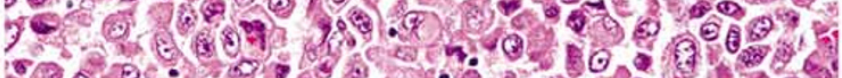

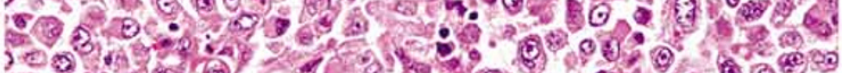
$30 \%$ (3)

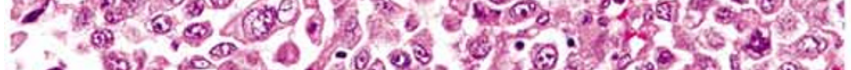

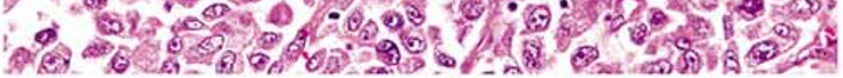
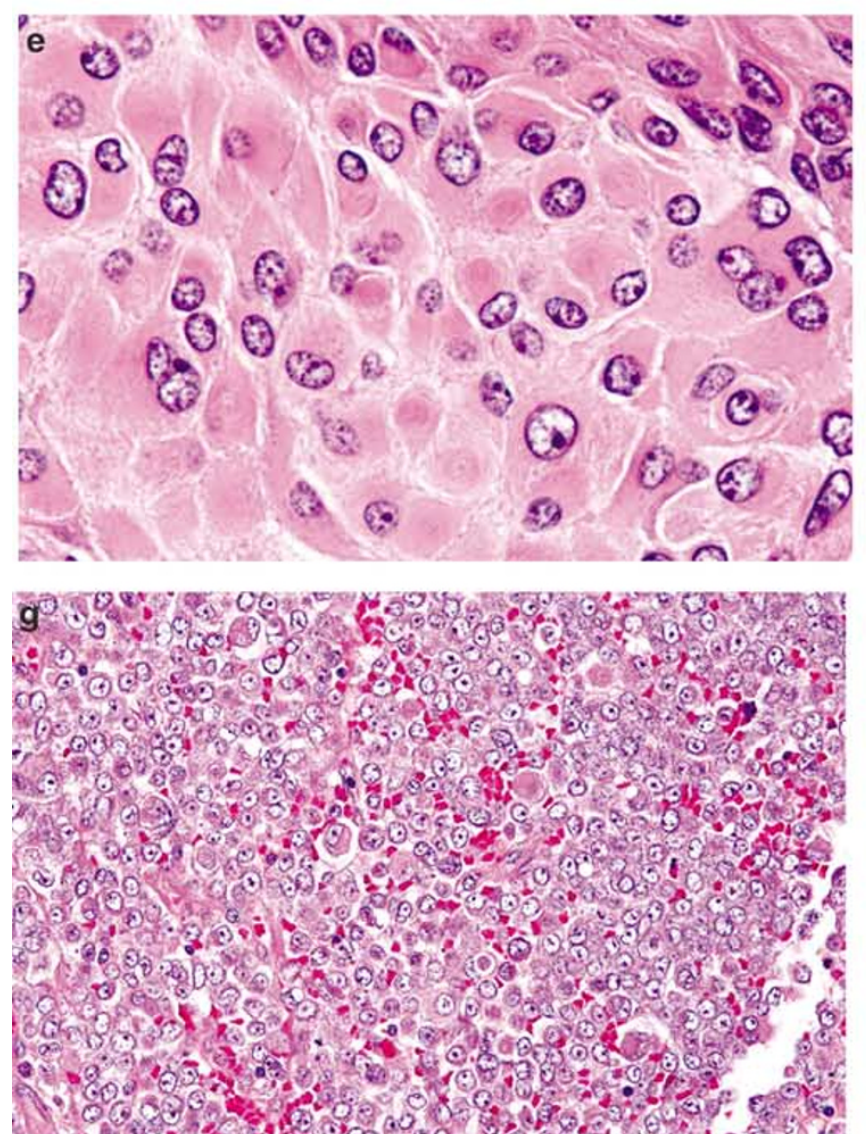

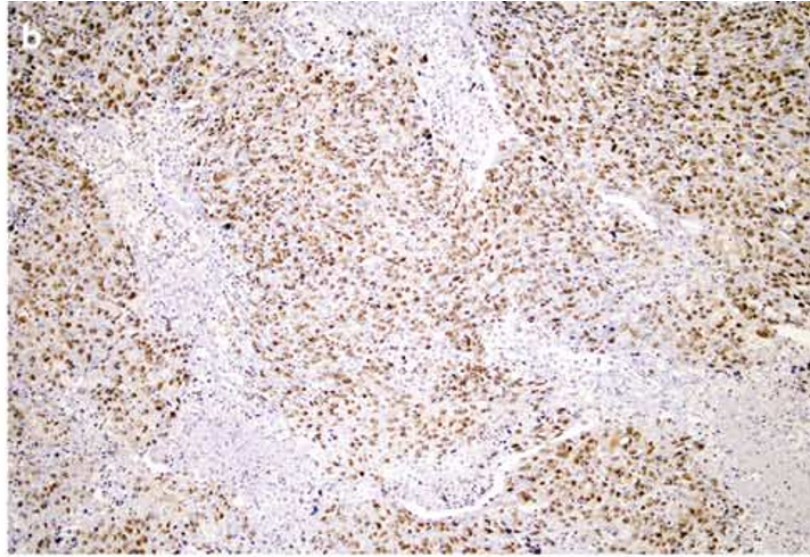

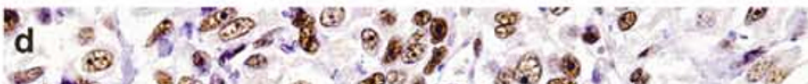

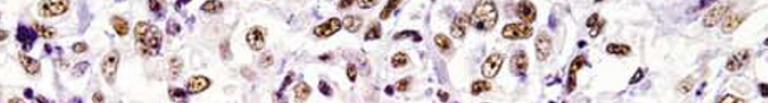

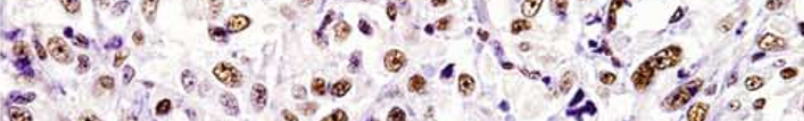

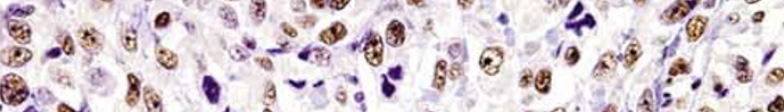

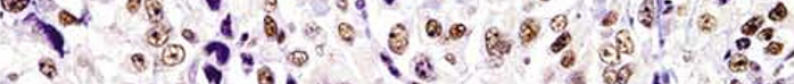

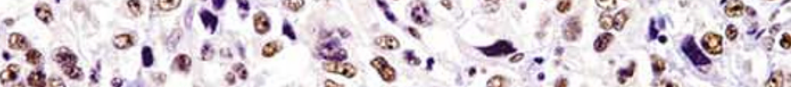

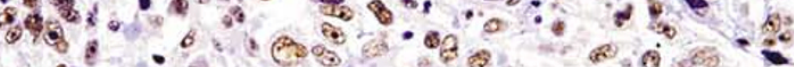

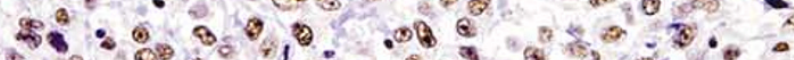

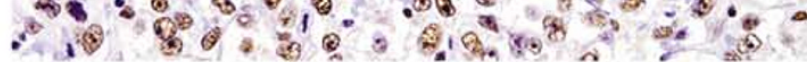

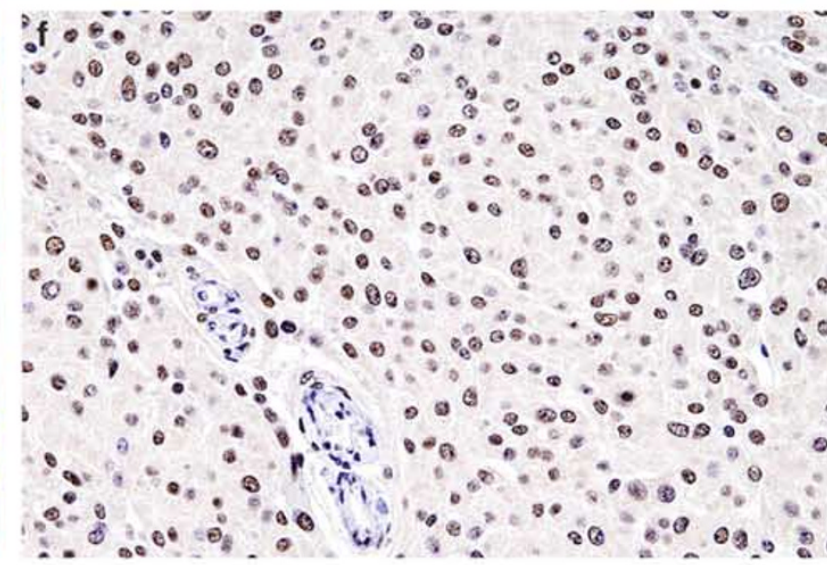

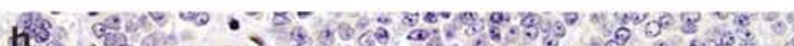

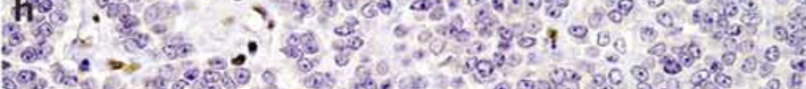

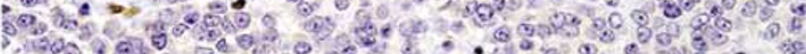

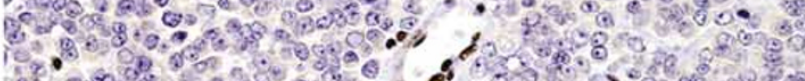

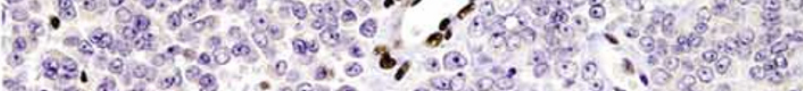

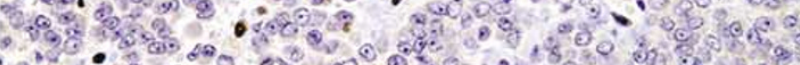

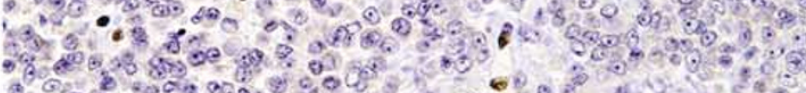

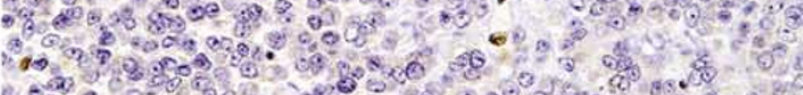

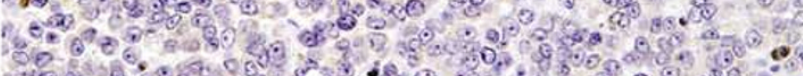

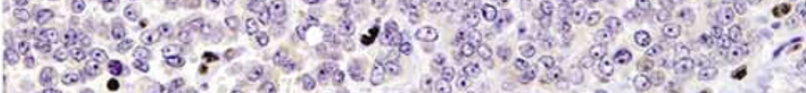
a. 

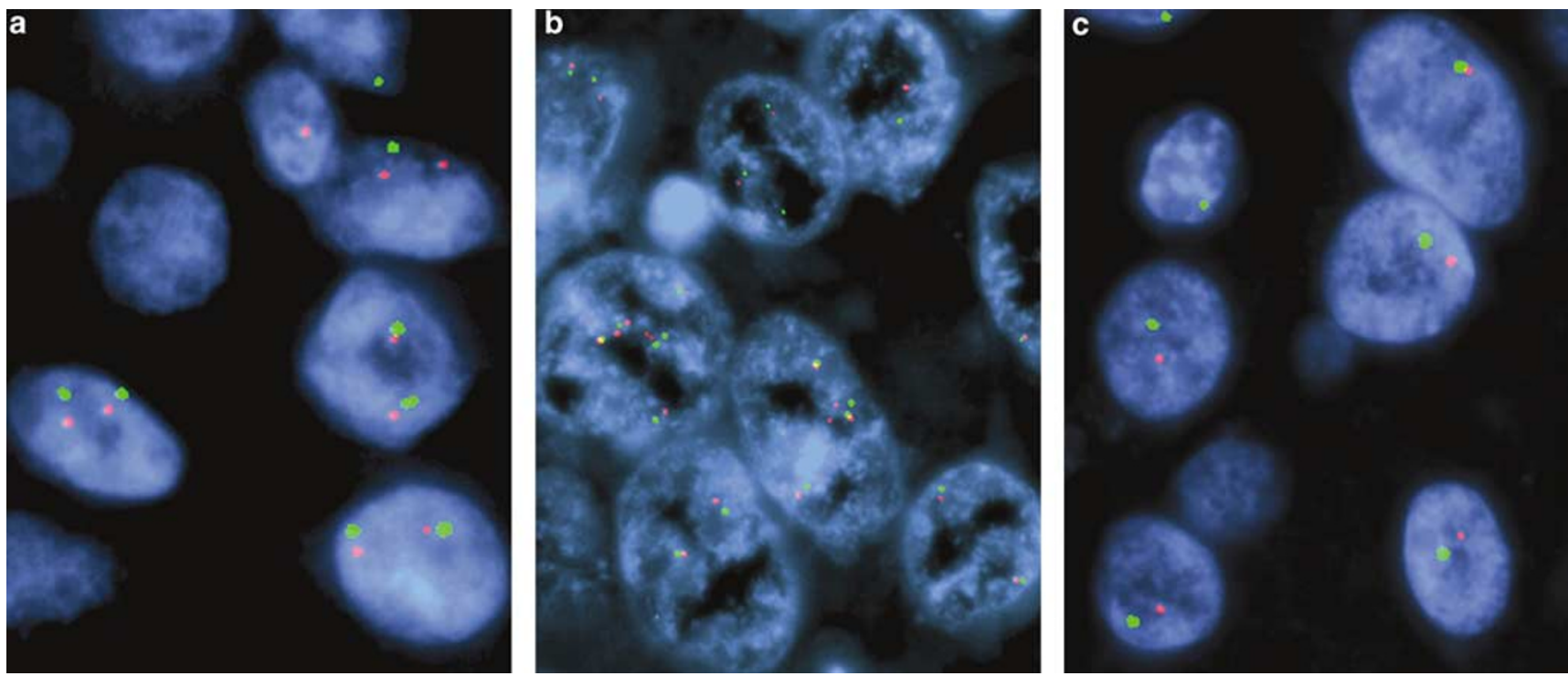

Figure 2 Representative FISH images from three cases of composite rhabdoid tumor showing normal 22q dosages with two green BCR and two red NF2 signals (a), polysomy 22q with $>2$ green and $>2$ red signals (b), and 22q deletion with one green and one red signal in most nuclei (c).

they are typically highly anaplastic tumors and the parent neoplasm is not always readily appreciable. Given the nearly uniformly poor prognosis, some have speculated that rhabdoid cells merely represent an end-stage phenotype, rather than a specific entity. However, recent data have shown that malignant rhabdoid tumor and atypical teratoid/rhabdoid tumor, in particular, share a characteristic genetic background with biallelic inactivation of the INI1/hSNF5 tumor suppressor gene on chromosome 22q11.2 in most cases. ${ }^{15-24} \mathrm{~A}$ congenital disseminated form has also been recognized and is often associated with mutations of the same gene.

Although its function remains to be fully elucidated, the INI1 protein product has been identified as a member of the SWI/SNF multiprotein complex involved in chromatin remodeling. ${ }^{22}$ It is ubiquitously expressed in non-neoplastic nuclei and therefore, intratumoral endothelial cells and lymphocytes provide a useful internal control for immunohistochemical studies. An antibody applicable to routinely processed paraffin-embedded tissue has recently been developed and studies have shown uniform INI1 loss in malignant rhabdoid tumor and atypical teratoid/rhabdoid tumor. ${ }^{23,24}$ In the current study, we found that in contrast, it is retained in the great majority of composite rhabdoid tumors, including those arising within neoplasms that also commonly harbor $22 \mathrm{q}$ deletions, such as meningiomas. The latter is particularly relevant given that INI1 mutations have been recently reported in a small subset of meningiomas..$^{37,38}$ Given that this gene was not inactivated in our cases, it is likely that the primary target in the subset of rhabdoid meningiomas with $22 \mathrm{q}$ deletion is the more telomeric NF2 gene on $22 \mathrm{q} 12$, just as it is in classic meningiomas.

Recent immunohistochemical studies of malignant rhabdoid tumors and atypical teratoid/rhabdoid tumors have shown that there was uniform loss of INI1 throughout the entire tumor, both in rhabdoid and nonrhabdoid tumor cells. ${ }^{23,24}$ Similarly, composite rhabdoid tumors in the current study tended to retain expression throughout the tumor, regardless of cytologic features. The cumulative data therefore argue against the notion that there is a common molecular denominator involved in the formation of all rhabdoid cells and provide further support for the existence of both a distinct 'entity' and a secondary 'phenotype'. Nevertheless, the possibility of rare composite rhabdoid tumors with loss of expression is intriguing, as in our case of the retroperitoneal leiomyosarcoma in which nearly the entire tumor was composed of epithelioid and rhabdoid cells and the diagnosis was based on smooth muscle differentiation by electron microscopy. The 29-year-old patient was an unlikely candidate for a diagnosis of malignant rhabdoid tumor, although not impossible, since we and others have encountered rare examples in adult patients. ${ }^{20,39-50}$ Lastly, INI1/hSNF5 mutation analysis was also attempted on this case, but the DNA did not amplify well (data not shown). In a recent study of pediatric CNS tumors, there was one case of an oligodendroglioma with loss of expression and a second case of mixed oligoastrocytoma with focal loss. $^{23}$ Similarly, a study of soft-tissue tumors revealed a few examples of synovial and epithelioid sarcomas with either weak or focal immunoreactivity. ${ }^{24}$ Therefore, the possibility that INI1 is rarely involved in other tumor types must be considered 
and would not be entirely surprising, given that most tumor suppressors are not absolutely specific to any single tumor type. Additional studies are needed to explore this possibility further. In any case, our data strongly argue that in the appropriate context, loss of INI1 expression is diagnostic of malignant rhabdoid tumor or atypical teratoid/ rhabdoid tumor.

In summary, INI1 immunohistochemistry represents the most useful ancillary technique currently available for resolving the differential diagnosis of malignant rhabdoid tumor or atypical teratoid/ rhabdoid tumor versus composite rhabdoid tumor, given that immunohistochemistry is much more widely available than FISH and both the sensitivity and specificity of this technique are considerably higher. Since neither technique is $100 \%$ accurate in all scenarios though, it remains important to consider all the relevant clinical, immunohistochemical, and genetic data (eg FISH, quantitative PCR, mutation analysis, etc) in complex cases.

\section{Acknowledgements}

This work was supported in part by the NIH Grant CA46274 (JAB).

\section{References}

1 Beckwith JB, Palmer NF. Histopathology and prognosis of Wilms tumors: Results from the First National Wilms' Tumor Study. Cancer 1978;41: 1937-1948.

2 Weeks DA, Beckwith B, Mierau GW, et al. Rhabdoid tumor of kidney. A report of 111 cases from the National Wilms' Tumor Study pathology center. Am J Surg Pathol 1989;13:439-458.

3 Weeks DA, Beckwith JB, Mierau GW. Rhabdoid tumor. An entity or a phenotype? Arch Pathol Lab Med 1989;113:113-114.

4 Ogino S, Ro JY, Redline RW. Malignant rhabdoid tumor: A phenotype? An entity?-a controversy revisited. Adv Anat Pathol 2000;7:181-190.

5 Parham DM, Weeks DA, Beckwith JB. The clinicopathologic spectrum of putative extrarenal rhabdoid tumors. Am J Surg Pathol 1994;18:1010-1029.

6 Wick MR, Ritter JH, Dehner LP. Malignant rhabdoid tumors: a clinicopathologic review and conceptual discussion. Semin Diagn Pathol 1995;12:233-248.

7 Fuller CE, Pfeifer J, Humphrey P, et al. Chromosome 22q dosage in composite extrarenal rhabdoid tumors: clonal evolution or a phenotypic mimic? Hum Pathol 2001;32:1102-1108.

8 Vujanic GM, Sandstedt B, Harms D, et al. Rhabdoid tumour of the kidney: a clinicopathological study of 22 patients from the International Society of Paediatric Oncology (SIOP) nephroblastoma file. Histopathology 1996;28:333-340.

9 Rorke LB, Packer RJ, Biegel JA. Central nervous system atypical teratoid/rhabdoid tumors of infancy and childhood: definition of an entity. J Neurosurg 1996; 85:56-65.
10 Burger PC, Yu I-T, Tihan T, et al. Atypical teratoid/ rhabdoid tumor of the central nervous system: a highly malignant tumor of infancy and childhood frequently mistaken for medulloblastoma-a pediatric oncology group study. Am J Surg Pathol 1998;22:1083-1092.

11 White FV, Dehner LP, Belchis DA, et al. Congenital disseminated malignant rhabdoid tumor: a distinct clinicopathologic entity demonstrating abnormalities of chromosome 22q11. Am J Surg Pathol 1999;23: 249-256.

12 Sajedi M, Wolff JEA, Egeler RM, et al. Congenital extrarenal non-central nervous system malignant rhabdoid tumor. J Pediatr Hematol Oncol 2002;24: 316-320.

13 Packer RJ, Biegel JA, Blaney S, et al. Atypical teratoid/ rhabdoid tumor of the central nervous system: report on workshop. J Pediatr Hematol Oncol 2002;24: 337-342.

14 Hilden JM, Meerbaum S, Burger P, et al. Central nervous system atypical teratoid/rhabdoid tumor: results of therapy in children enrolled in a registry. J Clin Oncol 2004;22:2877-2884.

15 Versteege I, Sevenet N, Lange J, et al. Truncating mutation of hSNF5/INI1 in aggressive paediatric cancer. Nature 1998;394:203-206.

16 Biegel JA, Zhou JY, Rorke LB, et al. Germ-line and acquired mutations of INI1 in atypical teratoid and rhabdoid tumors. Cancer Res 1999;59:74-79.

17 Sevenet N, Lellouch-Tubiana A, Schofield D, et al. Spectrum of hSNF5/INI1 somatic mutations in human cancer and genotype-phenotype correlations. Hum Mol Genet 1999;8:2359-2368.

18 Sevenet N, Sheridan E, Amram D, et al. Constitutional mutations of the hSNF5/INI1 gene predispose to a variety of cancers. Am J Hum Genet 1999;65: 1342-1348.

19 Rousseau-Merck MF, Versteege I, Legrand I, et al. hSNF5/INI1 inactivation is mainly associated with homozygous deletions and mitotic recombinations in rhabdoid tumors. Cancer Res 1999;59: 3152-3156.

20 Bruch LA, Hill DA, Cai DX, et al. A role for fluorescence in situ hybridization detection of chromosome $22 \mathrm{q}$ dosage in distinguishing atypical teratoid/rhabdoid tumors from medulloblastoma/central primitive neuroectodermal tumors. Hum Pathol 2001; 32:156-162.

21 Zhang F, Tan L, Wainwright LM, et al. No evidence for hypermethylation of the hSNF5/INI1 promoter in pediatric rhabdoid tumors. Genes Chromosomes Cancer 2002;34:398-405.

22 Biegel JA, Kalpana G, Knudsen ES, et al. The role of INI1 and the SWI/SNF complex in the development of rhabdoid tumors: meeting summary from the workshop on childhood atypical teratoid/rhabdoid tumors. Cancer Res 2002;62:323-328.

23 Judkins AR, Mauger J, Rorke L, et al. Immunohistochemical analysis of hSNF5/INI1 in pediatric CNS neoplasms. Am J Surg Pathol 2004;28:644-650.

24 Hoot AC, Russo P, Judkins AR, et al. Immunohistochemical analysis of the hSNF5/INI1 distinguishes renal and extra-renal malignant rhabdoid tumors from other pediatric soft tissue tumors. Am J Surg Pathol 2004;28:1485-1491.

25 Kepes JJ, Moral LA, Wilkinson SB, et al. Rhabdoid transformation of tumor cells in meningiomas: a histologic indication of increased proliferative activity. 
Report of four cases. Am J Surg Pathol 1998;22: 231-238.

26 Perry A, Scheithauer BW, Stafford SL, et al. 'Rhabdoid' meningioma: an aggressive variant. Am J Surg Pathol 1998;22:1482-1490.

27 Gokden N, Nappi O, Swanson PE, et al. Renal cell carcinoma with rhabdoid features. Am J Surg Pathol 2000;24:1329-1338.

28 Oshiro Y, Shiratsuchi H, Yoshinao O, et al. Rhabdoid features in leiomyosarcoma of soft tissue: with special reference to aggressive behavior. Mod Pathol 2000;13: 1211-1218.

29 Knapik J, Yachnis AT, Ripley D, et al. Aggressive uterine sarcoma with rhabdoid features: diagnosis by peritoneal fluid cytology and absence of INI1 gene mutation. Hum Pathol 2001;32:884-886.

30 Shimazaki H, Aida S, Sato M, et al. Lung carcinoma with rhabdoid cells: a clinicopathological study and survival analysis of 14 cases. Histopathology 2001;38: 425-434.

31 Wyatt-Ashmead J, Kleinschmidt-DeMasters BK, Hill $\mathrm{DA}$, et al. Rhabdoid glioblastoma. Clin Neuropathol 2001;20:248-255.

32 Levine PH, Mittal K. Rhabdoid epithelioid leiomyosarcoma of the uterine corpus: a case report and literature review. Int J Surg Pathol 2002;10:231-236.

33 Kuroiwa K, Kinoshita Y, Shiratsuchi H, et al. Renal cell carcinoma with rhabdoid features: an aggressive neoplasm. Histopathology 2002;41:538-548.

34 Abbott JJ, Amirkhan RH, Hoang MP. Malignant melanoma with a rhabdoid phenotype: histologic, immunohistochemical, and ultrastructural study of a case and review of literature. Arch Pathol Lab Med 2004;128:686-688.

35 Medjkane S, Novikov E, Versteege I, et al. The tumor suppressor hSNF5/INI1 modulates cell growth and actin cytoskeleton organization. Cancer Res 2004;64: 3406-3413.

36 Shiratsuchi H, Saito T, Sakamoto A, et al. Mutation analysis of human cytokeratin 8 gene in malignant rhabdoid tumor: a possible association with intracytoplasmic inclusion body formation. Mod Pathol 2002;15:146-153.

37 Schmitz U, Mueller W, Weber M, et al. INI1 mutations in meningiomas at a potential hotspot in exon 9 . Br J Cancer 2001;84:199-201.
38 Rieske P, Zakrzewska M, Piaskowski S, et al. Molecular heterogeneity of meningioma with INI1 mutation. Mol Pathol 2003;56:299-301.

39 Horn M, Schlote W, Lerch KD, et al. Malignant rhabdoid tumor: primary intracranial manifestation in an adult. Acta Neuropathol 1992;83:445-448.

40 Cossu A, Massarelli G, Manetto V, et al. Rhabdoid tumours of the central nervous system. Report of three cases with immunocytochemical and ultrastructural findings. Virchows Archiv A Pathol Anat 1995;422: 81-85.

41 Fisher BJ, Siddiqui J, Macdonald D, et al. Malignant rhabdoid tumor of brain: an aggressive clinical entity. Can J Neurol Sci 1996;23:257-263.

42 Ashraf R, Bentley RC, Awan AN, et al. Implantation metastasis of primary malignant rhabdoid tumor of the brain in an adult (one case report). Med Pediatr Oncol 1997;28:223-227.

43 Ben Taib NO, Nagy N, Salmon I, et al. Malignant rhabdoid tumors of the central nervous system. Report of a case. Neurochirurgie 1999;45:237-242.

44 Ogino S, Cohen ML, Abdul-Karim FW. Atypical teratoid/rhabdoid tumor of the CNS: cytopathology and immunohistochemistry of insulin-like growth factor II, insulin-like growth factor receptor type I, cathepsin D and ki-67. Mod Pathol 1999;12:379-385.

45 Arrazola J, Pedrosa I, Mendez, R, et al. Primary malignant rhabdoid tumour of the brain in an adult. Neuroradiology 2000;42:363-367.

46 Kuge A, Kayama T, Tsuchiya D, et al. Suprasellar primary malignant rhabdoid tumor in an adult: a case report. No Shinkei Geka 2000;28:351-358.

47 Lutterback J, Liegibel J, Koh D, et al. Atypical teratoid/ rhabdoid tumors in adult patients: case report and review of the literature. J Neuro-Oncol 2001;52:49-56.

48 Ohgaki M, Higuchi A, Chou $\mathrm{H}$, et al. An extrarenal malignant rhabdoid tumor suspected to originate from the mesentery in an adult: report of a case. Surg Today 2003;33:556-559.

49 Peng HQ, Stanek AE, Teichberg S, et al. Malignant rhabdoid tumor of the kidney in an adult: a case report and review of the literature. Arch Pathol Lab Med 2003;127:e371-e373.

50 Raisanen J, Biegel JA, Hatanpaa KJ, et al. Chromosome $22 \mathrm{q}$ deletions in adult atypical teratoid/rhabdoid tumors. Brain Pathol, in press. 